rapidly increased, and at length the eleventh dorsal vertebra became prominent, but no tumour could be felt, and there was no tenderness on percussion over the spine. He died about twelve months after I first saw him, and at the postmortem examination a small oxalate of lime calculus was found in his right kidney, and there was a tumour connected with the lower dorsal and first lumbar vertebræ, which was examined microscopically by Dr. Goodhart, and reported by him to be carcinoma.

Mr. Jacobson, writing on this subject, ${ }^{2}$ says, "the great difficulty which may arise in diagnosing between certain cases of spinal caries and renal calculus is not yet sufficiently recognised," and Mr. G. A. Wright ${ }^{3}$ has recorded a case in which, without any deformity or tenderness of the spine, nearly all the symptoms of a renal calculus were excited by deep suppuration (due to spinal caries), causing pressure upon the kidney.

Experience has taught me that it is not only in cases of disease but also of injury that mistakes in diagnosis are likely to be made between spinal and renal affections, and wrong treatment adopted accordingly.

11. Undetected Renal Calculus. - In two cases in which I have explored for stone and failed to find one, a calculus was present. Both these cases occurred before the exploration was so thoroughly carried out as now. One is the case now well known, which I published in the Med. Chir. Trans., vol. Ixviii, 1885 , in which I removed the kidney, with a stone imbedded in it, six months after the exploration. The other patient is a gentleman living in the north of England, whom I saw in consultation with Sir Henry Thompson and Mr. S. Mills and upon whom I operated on February 25th, 1888, assisted by Mr. S. Mills and Dr. Ayre Smith. I still hear constantly about, and occasionally see this gentleman, and I feel confident from the course, and present condition, of his case that there is a stone in his kidney.

12. No sufficient Cause discovered.-There are four of these cases on my list-two women and two men. One of the women was exceedingly neurotic, and I think her symptoms were due to that cause. She was frequently in the hospital under my charge after she was first recommended to me by Dr. Child, of Oxford. Her persistence in thinking she should never get well without an operation induced me at last to explore her kidney. In the other woman, aged 37 (the wife of a naval officer sent to me by Mr. Distin Maddick), there were very pronounced symptoms, and she had been under the care of Mr. Elliot Square, of Plymouth, who wrote that he had no doubt that there was renal calculus with cystic dilatation, and that he had advised an operation. Pain, tenderness, hæmaturia, pyuria, and oxalate crystals in the urine were all present, and she had passed pieces of mortar-like stone. Her bladder had been twice examined by Mr. Square. On May 23rd, 1891, the left kidney was explored, and a small hard lump the size of a horse bean was found in the upper part of it ; this was incised, and proved to be a cyst. The interior of the kidney was examined through the usual incision in the convex border, but nothing further was detected. She left the hospital improved on July 17th, 1891.

The two men were aged 24 and 20 respectively; both had nephralgia and hæmaturia, with pain shooting towards the bladder and along the penis. In neither was anything abnormal found in the kidney.

Such is the record of these 28 cases. If we analyse them, we find that in 20 cases some morbid condition of the kidney or the perinephric tissue was found. In another case nothing was discovered at the first exploration, but a stone was present, and was subsequently removed with the kidney. In the 22nd case, the stone was not found, though it is no doubt there, and, considering the present state of the patient, ought to be again searched for now that the method of search is so much more perfect. In 6 cases nothing at all abnormal was found in the kidney or its surroundings, but in 2 of them organic disease affected the prostate and other parts of the lower urinary apparatus, in 1 the symptoms were excited by a gastric ulcer, and in the other 3 no explanation of their symptoms is afforded by anything at present known about the patients.

In 3 of the cases in which some pathological condition was 2 The Operations of Surgery, Jacobson, 2nd edition, p. 730. 3 Mied. Chron., No. vi, p. 642 . found there was grave disease in other organs as well. In 1 a movable kidney probably caused the whole of the symptoms; in another the mobility of the kidney explained only part of the symptoms, the rest being caused by a calculus lower down. In 14 of the cases complete cure followed the operation; and in another life was prolonged for nearly three years, and great relief from suffering secured by the operation.

It is only twelve years since nephrolithotomy and the search for stones in the kidney was introduced into practice, but I feel that it is time now to publish the details of my experience in those cases which may cynically be styled " the fruitless:search after stones." But let it be remembered that in 21 of these "searches" disease of some kind of, or around the kidney was discovered by the exploration; that in 15 cases restoration to active life, with complete recovery in 14 , was the result. That many of these patients were cured after years of serious suffering rendering them incapable of following their ordinary lives, that at least two others with our present experience would have been cured, and that in no instance did any ill consequence happen from an exploratory operation.

Let these results be compared with those of exploratory laparotomy, exploratory trephining, digital examination of the bladder, the aspiration of tumours, and tapping the pleural sacs. Let it be remembered too that these renal explorations have been made in the first decade in the history of the operation; that during that time our methods of procedure have been vastly improved, and then I think it will be allowed that the prospects of the operation in the immediate future will be even better, and the results even more satisfactory and encouraging than those I have now laid before you.

The difficulties in diagnosis of renal calculus are many, and at present do not seem likely to be overcome, or materially lessened; and, therefore, taking into consideration the almost absolute immunity from danger attending the operation; physicians ought to recommend more frequently and at a much earlier period, an exploration of the kidney to assist in clearing up the diagnosis of doubtful cases.

\section{REMARKS ON THE TREATMENT OF PLEURISY.}

EDWIN RICKARDS, M.B.OxoN., Physician to the General Hospital, Birmingham.

Pledrisy is a disease which arises under various conditions, which, to some extent, determine its treatment. The limited time at my disposal prevents my particularising the modification of treatment requisite for all the various kinds 0 pleurisy arranged on an etiological basis; nor can I suggest any classification of pleurisies on such a basis which would not involve much difference of opinion.

The differentiation of pleurisies into primary and secondary is not accepted by all authorities. There are those who doubt if inflammation of the pleural membrane is ever a primary condition, and hold that that membrane enjoys the same immunity from idiopathic inflammation as do other serous membranes, as the peritoneum and pericardium.

There is now, I believe, a general consensus of opinion that in most cases of pleurisy the disorder is a secondary onesecondary to disease of some neighbouring organ or adjoining part, or to some dyscrasic condition of the blood. Besides, in view of treatment, I think that a pathological differentia tion of pleurisies is even better than an etiological one. Otten in secondary pleurisy we have to commence treatment while in the dark as to its primary cause. I shall therefore, for the purpose of this address, speak of pleurisy as being of three kinds :-

1 Read before the Birmingham and Midland Counties Branch of the British Sedical Association. 
1. Dry pleurisy, where there is little or no effusion into the pleural sac.

2. Effusive pleurisy, where there is effusion which is fibroserous and clear.

3. Empyema, where there is purulent effusion.

Dry pleurisy often does not give rise to symptoms which lead to its recognition, and cohesion of the two layers of the pleural membrane is the only post-mortem evidence of its preexistence. It is admittedly a secondary affection. In lung disease pain is probably always an expression of pleurisy, which is often overshadowed by the substantive disease, and escapes treatment. Dry pleurisy in lung diseases, by sealing the lung to the chest wall, may prevent any escape of morbid products from the lung into the pleural sac, to the well-being of the individual. In dry pleurisy pain is in most cases the symptom which leads to its diagnosis and most calls for help. Our present treatment of dry pleurisy differs from that of former times chiefly in our having given up heroic remedies. The disease being a conservative one, we must not, I think, be too busy with our curative agents-we may safely trust largely to rest in bed. For the relief of pain we have discarded the lancet, and rely on a hot poultice or a few leeches locally, or, still better-where there is no contra-indication to the drug - a dose of opium.

Effusive pleurisy usually sets in with definite symptoms; its pyrexia is accompanied by an outpouring of fluid into the pleural sac, and it is the accumulation of this fluid which causes all our anxiety; without it there would be no danger to life, either from the immediate or remote effects of pleurisy; without it pleurisy would be a disease of short duration.

The question here arises, Can we, by treatment, prevent effusion in a pleurisy which, without treatment, would be effusive? I think not. With this object in view, resort has been had to bleeding, blistering, poultices, purgatives, diuretics, diaphoretics, mercury, opium, aconite, quinine, and the more specific antipyretics, but, as far as my experience goes, without avail. I am ready to admit that, in some cases of acute pleurisy, as in most acute inflammatory affections, a calomel purge at the onset does good by freeing the secretions and lowering arterial tension, and I have sometimes thought that in the early stage of acute pleurisy I have seen the severity and duration of the febrile stage modified by a few small doses of calomel and opium, but I have never been able to satisfy myself that any method of treatment has prevented effusion. Then comes the question, Have we any means of promoting the natural absorption of the effusion? Beyond keeping our patient in bed, and improving the general health, I think we have not. Blisters, iodine applications, dry diet, diaphoretics, mercury, and especially diuretics, have long enjoyed-I consider undeservedly - a great repute for this purpose. Diuretics are, in my opinion, absolutely useless, and, worse than that, are harmful. As commonly prescribed, they do not produce diuresis, but they always lower the vitality of the individual.

We can now, with impunity, artificially remove the effusion by aspiration or siphonage. The exact time when such operative procedure should be practised will for some time to come be an unsolved problem. Aspiration and siphonage are used for the same purpose, namely, the extraction of fluid from the chest without the admission of air. The siphon has the advantages of exerting little force and of removing the fluid very gradually; but, on the other hand, the aspirator not infrequently succeeds in extracting fluid when the siphon fails. For aspiration or siphonage I always use a trocar and cannula; for the latter operation I employ a No. 7 French gauge; for the former, a smaller one, No. 6 French gauge; but before resorting to either method I always satisfy myself by an exploring syringe that there is fluid at the spot for operation, and-when I can choose the spot-I select the ninth interspace at the back, below the angle of the scapula.

It may be said that we practice aspiration for four objects. One being the prevention or relief of urgent symptoms, to prevent death from the effects of the pressure of rapid and excessive effusion. Many are the laws which have been laid down as to the time for operating under these circumstances ; some have been founded on the extent of the fluid dulness, some on the quantity of fluid in the chest, some on the amount of the displacement of the heart, others on symptoms themselves of which dyspncea and lividity are always the most prominent ones, and are due-for the most part at least - to the pressure of the effusion on the heart and its large vessels. The extent of fluid-dulness may be great, even up to the clavicle, and yet, provided the patient be kept in bed need cause no anxiety; whereas with a much smaller extent of fluid dulness there may be alarming symptoms. The amount of fluid in the chest must always at best be but a speculation. There may be danger without much displacement of the heart; the most reliable guide to my mind is the symptom dyspnœa. Dyspnca gives timely warning of danger, and when it assumes the form of orthopnœa, delay in the removal of some fluid is dangerous. At one operation I generally remove about two pints of fluid, unless-before that amount has been extracted-troublesome cough or cardiac distress supervene, or unless the fluid, at first clear, should become decidedly bloody. These are indications for desisting from aspiration.

Our second object in aspiration is to shorten the duration of the disease. No one will dispute that in many cases of pleuritic effusion the fluid will entirely disappear, and the patient will get well if kept in bed without special treatment in the course of six weeks. But if we can by aspiration do in twenty minutes what the natural process of absorption sometimes takes as many days to effect, surely by a harmless and trifling operation we are bound to save our patients weeks of illness. But more than that; we never can tell till late in the case-and sometimes till too late-whether the fluid will be removed by absorption in a few weeks or whether it will be absorbed at all. The question here arises, When should we operate for the object now under consideration? My answer is, Not until the febrile movement has ceased, unless that movement should continue more than a fortnight.

In 1873 Dr. Castiaux, a Parisian practitioner, advocated the removal of as much fluid as possible as soon as it is detected. He urged that the earlier and the more complete its removal the less likelihood there was of its recurrence; and more than that, he maintained that its removal extinguished the initial fever and ended the disease. Moutard-Martin and others supported him in these views. My experience does not enable me to go all the way with Castiaux. By operating in the early stage of the fever I have found the pyrexia never extinguished and rarely reduced, and reaccumulation of fluid universal. I have, however, seen cessation of the fever on aspirating when the febrile stage has been unusually prolonged, and I have several times used the operation for this purpose successfully. There is no doubt in my mind that when the febrile stage is over, the sooner the fluid is removed the less likely it is to reaccumulate and the more rapid the recovery of the patient. For our present object I withdraw as much fluid as I can, desisting, however, if troublesome cough, cardiacdistress, or faintness should supervene, or if the fluid, at first clear, should become decidedly blood-stained. If further aspirations are required, I allow an interval of three days between them. Operating on the fall of the fever, more than a second aspiration is rarely requisite; one frequently suffices. Often after aspiration dulness on percussion at the back has. led me to think that there was reaccumulation of fluid, when the exploring needle proved that such was not the case.

Our third object in artificial removal of fluid is to prevent a pleuritic effusion from becoming chronic. Chronic pleuriticeffusion, which I define as a pleuritic effusion which, in spite of treatment, persists for three or four months, is 8 grave disorder, more so, to my mind, than empyema; repeated aspirations are followed by repeated reaccumulations. The patients, broken-hearted, go from doctor to doctor, from hospital to hospital; some get well in the long run, but the run is a very long one; some die of phthisis; in some theeffusion becomes purulent; in some the effusion is permanent. Some of my cases have gone from my observation uncured. One man recently under my care, who had had pleuritic effusion for four months, and had been submitted to treatment (short of aspiration), was cured in three months by rest in bed, a generous diet, tonics, and repeated aspirations. It seems to me probable that, as time goes on, we shall treat these cases as we do empyema, by pleurotomy.

Chronic pleuritic effusion is, I believe, generally the result: of allowing those who have had acute effusive pleurisy to. walk about before the fluid has entirely disappeared. Fluid. in the pleural cavity is always slow to go and often remains 
stationary for an indefinite length of time if the patient is walking about: and it is difficult to impress upon those who are getting well of acute effusive pleurisy, when the fever has subsided and they feel well, the importance of staying in bed until the fluid is quite gone.

Our fourth object in aspiration is to obviate the possibility of the collapsed lung becoming inexpansible, clear pleuritic fluid remaining in the chest a long time, but a period of time varying in different cases may, like empyema, cause the lung to become permanently incapable of expansion. In one such case under my care, from the history it was judged that the chest had been full of fluid only six weeks. Such an effect in so short a time is, however, exceptional in pleuritic effusion where the fluid is fibrino-serous. Such cases are incurable; aspiration converts them into empyemata.

While effusive 'pleurisy usually sets in with definite symptoms, there is a kind which has been called latent pleurisy in which, when a patient first comes under observation, the chest is full of fluid. In such a case the pain and initial fever not being severe fail to attract the patient's attention to the chest, and it is only when the effusion causes dyspncea on exertion that medical aid is sought. Latent pleurisy is, in my opinion, very different from chronic pleurisy. The effusion in latent pleurisy will generally disappear in a few weeks if the patient be kept in bed, and by aspiration the cure may be effected in a much shorter time.

Pleuritic effusion is often found in women in the puerperal state and for months after parturition. It is readily cured by aspiration, but not infrequently two or three operations are required. I. do not here refer to the pleuritic effusion which is part of puerperal fever where the fluid is sometimes pus, and has to be treated accordingly. Pleuritic effusion in acute rheumatism is rapidly absorbed, and seldom requires artificial removal save in bad cases for the relief of urgent symptoms. The effusion of chronic Bright's disease is liable to return after removal; aspiration gives but temporary relief.

The effusion of early phthisis is very amenable to aspiration, but in that of advanced phthisis the advantage of operative interference is very questionable. We frequently find pleuritic effusion in persons with an hereditary tendency to phthisis, but without any physical signs of that disease in the chest. Early aspiration cures such cases rapidly and completely. Let me cite a case: A man, aged 27, of very consumptive appearance, came to me with fluid in his left chest; he had been ill three weeks; his father died of consumption; he alone remained of a family of seven, the others having died of consumption. I aspirated off three pints of fluid. Eight days after the operation, I explored the chest in several places without tapping fluid; he was practically well. I saw him six months afterwards; he was in rude health, stronger than he had been for years previous to this illness. Such a case is by no means of uncommon occurrence. As to the primary cause of pleurisy in such a case, I must confess I am at present in the dark, though the balance of probabilities seems to me to point to a crop of tubercles in the pleural membrane.

I shall not detain you long with the treatment of empyema; here drugs and aspiration are powerless-pleurotomy is allpowerful. There are cases on record of recovery when the disease has been left to Nature, where the pus has remained permanently as a cheesy or calcareous mass in the pleural sac, where the pus has escaped by a self-made opening through the chest wall or the lung. The literature of the subject contains instances of cures by aspiration, by single or repeated use of the trocar and cannula, but all these are exceptional cases, and may be regarded as curiosities.

When it is known that pus is in the chest, the sooner it is let out by free incision, the more rapid, the more complete, the more certain the recovery; but besides and beyond this, early operation diminishes the risk of the lung becoming permanently inexpansible; in other words, it lessens the probability of a curable case becoming an incurable one.

Our suspicions of empyema may be raised at the onset of a pleurisy; rigors, excessive and long-continued pain, a temperature above $102^{\circ} \mathrm{F}$., the association of the pleurisy with catarrhal pneurnonia, or acute nephritis, or scarlet fever, or septicæmia, fluid in the chest of a young child, point to empyema. Our suspicions are crystallised into a conviction by the supervention of hectic, or perhaps more properly, of septic fever. The exploring syringe, without risk and with little discomfort to the patient, can at any moment set all our doubts at rest.

An aspiration as a preliminary to pleurotomy has been advocated in view of the more gradual expansion of the lung and its partial adhesion to the chest wall before air enters the incised chest. But one is often baulked in aspiration or siphonage by the plugging of the cannula by caseous coagula. If this method be adopted I think the interval between the two operations should not exceed three days, for about that time a septic condition generally develops. I have more than once resorted to this plan when the pleural sac was very full of pus, but my experience on the matter is too small to be of any value.

And now a word about pleurotomy. When I can select the spot for the operation, when pus is not encapsuled, and when the lung is not adherent in places to the chest wall, I get the surgeon to make a single incision in the ninth interspace at the back below the angle of the scapula; but before he does so I always, without exception, prove by the exploring syringe that there is pus at that spot: such a procedure may save wounding the lung or the diaphragm if these should have become adherent to the chest wall in that region. When the chest has been opened what is wanted is some means which would permit the outflow of pus and prevent the ingress of air into the chest, both at the time of the operation and afterwards. After the operation by the usual method the ordinary dressings act in this way, but to a very limited extent. Recently I have adopted a plan which I will now briefly describe. As soon as the chest has been incised and the pus begins to flow and before air begins to enter the chest I place over the opening a piece of oil-silk, 8 inches square; this acts as a valve and allows the pus to escape from under it and admits no air. When the flow of pus has ceased, even on coughing, I place a layer of cotton wool over the oil-silk and fix it with an elastic bandage. This method does not prevent the use of a drainage tube, protected by a safety pin.

In dressing the case the following day, a fresh piece of oil-silk may be slid under the old one. If, on the patient's coughing, there should be no discharge from the opening or from the drainage tube (if one be used) the patient should be urged to stop breathing, the oil-silk and drainage tube should be quickly removed, the incision should be rapidly reopened with dressing forceps, and the oil-silk instantaneously reapplied.

Recoveries under this plan have been rapid. I think that if air can be excluded for twelve hours after the operation, and only a limited amount enters during the dressing, and none between the dressings, there is every inducement to the lung to expand ; whereas, if air goes freely in and out of the chest by the incision, as in the ordinary method, there is no encouragement to the lung to expand. If the lung cannot expand, my method and all methods are of no avail. I would here remark that while fluid is continuously flowing out from the pleural sac, no air enters.

If, after the chest has been opened for empyema, the lung cannot expand, the condition of things is most grave, the life of the individual is not worth more than two years' purchase. A patient with pyopneumothrax, an inexpansible lung, and a thoracic fistula may walk about, and even improve in general health ; but, as time goes on, ulceration of the surface of the lung by irritation sets up phthisis, and it may be said to be a contest between phthisis and albuminoid disease as to which shall destroy life. With such conditions, unless the pleural cavity can be entirely obliterated, a fatal issue is inevitable. In young children this is possible by displacement upwards of the diaphragm and abdominal viscera, and by a falling in of the chest wall which is pliable, and by granulation tissue; but in the adult with rigid walls this favourable result is not attainable. By Estlander's operation of removing ribs the cavity may be greatly diminished, and cases are on record where it has been obliterated and a cure the result ; but, as far as my observations have gone, the future of this operation is not full of promise.

Can we, I would ask, in a case of empyema prevent the lung from becoming inexpansible, and thereby reduce the mortality of the disease? I think we can. The inexpansibility of the lung is due to the postponement of pleurotomy, and would be obviated were that operation performed before the lung had become coated and bound down by false membranes. 